\title{
Natural history of hypercoagulability in patients undergoing coronary revascularization and effect of preoperative myocardial infarction
}

\author{
J. James Edelman, PhD, MBBS(Hons), ${ }^{\text {a,b,c }}$ Caroline J. Reddel, PhD, \\ Leonard Kritharides, PhD, FRACP, ${ }^{\mathrm{b}, \mathrm{c}, \mathrm{d}}$ Paul G. Bannon, PhD, FRACS, ${ }^{\mathrm{a}, \mathrm{b}}$ \\ John F. Fraser, PhD, MRCP, FRCA, FCICM, ${ }^{\mathrm{e}}$ Jennifer L. Curnow, PhD, FRACP, ${ }^{\mathrm{b}, \mathrm{c}, \mathrm{d}}$ and \\ Michael P. Vallely, PhD, FRACS ${ }^{\mathrm{a}, \mathrm{b}, \mathrm{f}}$
}

\begin{abstract}
Objectives: The balance between hyper- and hypocoagulable states is critical after coronary artery surgery both with (coronary artery bypass grafting $[\mathrm{CABG}]$ ) and without (off-pump coronary artery bypass [OPCAB]) cardiopulmonary bypass to prevent thrombotic or bleeding complications. We aimed to quantify novel parameters of coagulation, fibrinolysis, and overall hemostasis $\leq 6$ months after CABG and OPCAB and to determine the influences on these parameters.
\end{abstract}

\begin{abstract}
Methods: A total of 63 patients ( $30 \mathrm{CABG}, 33 \mathrm{OPCAB}$ ) had blood collected before and at various points $\leq 6$ months after surgery. Fibrin and fibrinolysis time curves were generated by measuring the absorption of 405 $\mathrm{nm}$ each minute for 100 minutes after the addition of tissue factor and tissue plasminogen activator to cellfree plasma. The parameters were compared with those from a group of healthy controls.
\end{abstract}

\begin{abstract}
Results: The patients' preoperative prothrombotic assay parameters were compared with those from healthy controls. Both CABG and OPCAB patients were hypercoagulable until at least day 10 after surgery, with elevation of fibrin generation (CABG, peak day $3,+28.9 \%$; OPCAB, peak day $1,+16.3 \%$ vs preoperative baseline) and impairment of fibrinolysis capacity (CABG, day $1,-58.4 \%$; OPCAB, day $1,-22.6 \%$ ). Surgical revascularization resulted in resolution of preoperative hypercoagulability by 6 months postoperatively. Patients with preoperative myocardial infarction (MI) had prolonged hypercoagulability after surgery that was most exaggerated after CABG (overall hemostatic potential day 5 , no MI, $+64.1 \%$ vs with $\mathrm{MI},+128.9 \%$ compared with baseline; $P=.013)$.
\end{abstract}

Conclusions: Patients will be vulnerable to thrombotic events for $\leq 6$ weeks after coronary surgery yet will have resolution of hypercoagulability by 6 months. Preoperative factors, such as MI, could require individualized management of thrombosis prophylaxis in the postoperative period. (J Thorac Cardiovasc Surg 2014;148:536-43)

Coronary artery bypass grafting, performed with (CABG) and without (off-pump coronary artery bypass [OPCAB]) cardiopulmonary bypass (CPB) causes a systemic inflammatory response and alterations to the normal mechanisms

From the Cardiothoracic Surgical Unit, ${ }^{\mathrm{a}}$ Royal Prince Alfred Hospital, The Baird Institute, Sydney, Australia; Sydney Medical School, ${ }^{\mathrm{b}}$ University of Sydney, Sydney, Australia; ANZAC Research Institute, ${ }^{c}$ Sydney, Australia; Concord Repatriation General Hospital, ${ }^{\mathrm{d}}$ Sydney, Australia; Critical Care Research Group, ${ }^{\mathrm{e}}$ The Prince Charles Hospital, Brisbane, Australia; and Australian School of Advanced Medicine, ${ }^{\mathrm{f}}$ Macquarie University, Sydney, Australia.

The Baird Institute received an unencumbered research grant from Medtronic.

Disclosures: Michael Vallely reports consulting fees from Medtronic. Paul Bannon reports consulting fees from Medtronic. Jennifer Curnow reports consulting fees from Boehringer Ingelheim and Novartis and lecture fees from Bayer and Boehringer Ingelheim. All other authors have nothing to disclose with regard to commercial support.

Received for publication Sept 1, 2013; revisions received Oct 2, 2013; accepted for publication Oct 11, 2013; available ahead of print Nov 25, 2013.

Address for reprints: J. James Edelman, PhD, MBBS(Hons), The Baird Institute, Suite 305, 100 Carillon Ave, Newtown, NSW 2042, Australia (E-mail: jjbedelman@gmail.com).

$0022-5223 / \$ 36.00$

Copyright (c) 2014 by The American Association for Thoracic Surgery

http://dx.doi.org/10.1016/j.jtcvs.2013.10.028 of hemostasis (ie, coagulation and fibrinolysis). ${ }^{1}$ A careful balance between coagulation and fibrinolysis is essential, particularly in the perioperative setting, when a prothrombotic state can render a patient prone to bypass graft failure, myocardial infarction (MI), stroke, or other venous or arterial thromboembolism, and a hypocoagulable state can result in life-threatening bleeding.

Thrombin generation during CPB has traditionally been thought to result primarily from exposure of blood to the nonendothelialized surface of the circuit, with activation of the intrinsic pathway of coagulation. More recently, the primary mechanism has been shown to involve generation of the VIIa/tissue factor (tenase) complex as a result of the surgical injury. ${ }^{2,3}$ The activation of hemostasis during $\mathrm{CABG}$ and OPCAB is complex and involves tissue factormediated activation of coagulation, fibrinolysis, inflammation, and platelets. ${ }^{4}$ Coronary revascularization without the use of CPB has been performed by some surgeons in the hope of reducing the morbidity associated with systemic inflammation ${ }^{5}$ and reduce the stroke risk associated with manipulation of the ascending aorta. ${ }^{6}$ 

Abbreviations and Acronyms
$\mathrm{CABG}=$ coronary artery bypass grafting
$\mathrm{CAD}=$ coronary artery disease
$\mathrm{CPB}=$ cardiopulmonary bypass
OCP $=$ overall coagulation potential
OFP $=$ overall fibrinolytic potential
OHP = overall hemostatic potential
$\mathrm{OPCAB}=$ off-pump coronary artery bypass
PAI-1 = plasminogen activator inhibitor 1
TXA $=$ tranexamic acid

Investigations of the indirect markers of fibrin generation (prothrombin fragments $1+2$, thrombin-antithrombin complex) and fibrinolysis (D-dimer) have suggested that both OP$\mathrm{CAB}$ and $\mathrm{CABG}$ are associated with a prothrombotic state that can persist for $\geq 1$ month after surgery. ${ }^{7,8}$ Whether the changes will be sustained beyond this time, and how the preoperative comorbidities modulate the response to surgery, are unknown. Preoperative MI increases the risk of morbidity and mortality after CABG; the risk of stroke with recent MI, in particular, is increased. ${ }^{9-11}$ MI disturbs the normal balance of coagulation and fibrinolysis ${ }^{12}$ - however, the possibility that an MI in the period immediately before surgery influences hemostasis, and, thus, thrombotic complications, after surgery has not been investigated.

Few clinical tests are routinely available to detect the states of hypercoagulability. The overall hemostatic potential $(\mathrm{OHP})$ is a global assay that quantifies the ex vivo potential for coagulation and fibrinolysis by generation of fibrin time curves. The OHP is able to define a hypercoagulable profile in a number of clinical prothrombotic states-pregnancy, unprovoked venous and arterial thromboembolism, and autoimmune conditions. ${ }^{13}$ More recently, impairment of fibrinolysis activity and enhancement of fibrin clot formation as identified by the OHP assay have been associated with MI. ${ }^{12}$

In the present study, we used the OHP assay to investigate the changes in coagulation and fibrinolysis after $\mathrm{CABG}$ and $\mathrm{OPCAB}$. We extended these observations from the preoperative period to 6 months and identify the preoperative MI as an independent contributor to the coagulation response to surgery.

\section{METHODS}

A total of 63 patients undergoing surgical coronary revascularization from 2010 to 2011 were invited to participate. Patients who were receiving therapeutic anticoagulation (with unfractionated/low-molecular-weight heparin or warfarin) immediately before surgery were not included. All patients provided written consent, and the Sydney Local Health Network Human Research Ethics Committee approved the present study (X09-0322).

\section{Surgical Revascularization}

The anesthetic regimen was standardized. The patients were premedicated with a benzodiazepine and opiate. Anesthesia was induced with propofol, fentanyl, and a nondepolarizing muscle relaxant and was maintained with a volatile agent. Antibiotics were administered before the induction of anesthesia. Aspirin was routinely ceased in CABG patients 7 days before surgery and continued to surgery in OPCAB patients. Variations to this occurred on clinical grounds (eg, high-risk coronary disease, bleeding risk). All patients were advised to cease medications, other than aspirin, known to influence coagulation (eg, fish oil, nonsteroidal antiinflammatory agents) 7 days before surgery.

\section{Cardiopulmonary Bypass}

Patients undergoing $\mathrm{CABG}$ with $\mathrm{CPB}$ received systemic heparinization (400 IU $/ \mathrm{kg}$ ) to maintain an activated clotting time of $>400$ seconds. At the conclusion of CPB, heparin was reversed with protamine $(1 \mathrm{mg} / 100 \mathrm{IU}$ of heparin given, then additional as required for an activated clotting time within normal limits). CPB was performed with mild to moderate hypothermia $\left(30^{\circ} \mathrm{C}-32^{\circ} \mathrm{C}\right)$. The circuit consisted of a roller pump (Jostra Medizintechnik AG, Hirrlingen, Germany), membrane oxygenator (Capiox SX18; Terumo, Tokyo, Japan) and polyvinyl chloride circuit tubing (Maquet, Rastatt, Germany) primed with $2500 \mathrm{~mL}$ isotonic compound lactate solution (Hartmann's solution; Baxter Healthcare, Sydney, Australia) and $10,000 \mathrm{U}$ heparin. Pericardial blood was salvaged using cardiotomy suction. Patients were routinely given the antifibrinolytic agent, tranexamic acid (TXA; $1.5 \mathrm{~g}$ loading dose, then $1 \mathrm{~g} / \mathrm{h}$ until the conclusion of CPB).

Antegrade, cold cardioplegia solution (St Thomas' solution) was used to arrest the heart in diastole. Rewarming began as the last distal anastomosis was fashioned, and separation from $\mathrm{CPB}$ occurred once the grafts were complete and the patients were normothermic $\left(>36.5^{\circ} \mathrm{C}\right)$. A combination of left and right internal thoracic artery and saphenous vein grafts were used as conduits.

\section{OPCAB Grafting}

Systemic heparinization (200-300 IU/kg) was used to maintain an activated clotting time of $>350$ seconds and was reversed with protamine at the conclusion of the case. Normothermia was maintained throughout. Patients were administered a low to mid-dose of milrinone $(0.2-0.4 \mu \mathrm{g} / \mathrm{kg} / \mathrm{min})$, without a loading dose, for arterial graft vasodilation. Milrinone was ceased after 24 hours.

TXA $(1 \mathrm{~g})$ was given to 9 of the 33 OPCAB patients. The decision to administer TXA was determined by 1 clinician's preference. Cell salvage was used in all cases. Myocardial stabilization was achieved with a stabilizing device (Medtronic Octopus; Medtronic, Minneapolis, Minn), and Silastic shunts were used for distal vessel perfusion to enable a bloodless field and prevent technical errors during the anastomosis.

\section{Postoperative Anticoagulation}

Deep vein thrombosis prophylaxis (heparin 5000 IU given subcutaneously, twice daily) was begun on the evening of the first postoperative day and continued until discharge from the hospital. Aspirin was begun on day 1 for all patients and continued indefinitely. The patients were discharged from intensive care when satisfactory cardiovascular, respiratory, and neurologic parameters had been met.

\section{Blood Sampling and Storage}

Blood samples were collected on the morning before surgery and 1,3,5, and 10 days, 6 weeks, and 6 months after surgery. Blood was collected into $0.109 \mathrm{M}$ sodium citrate BD Vacutainer tubes (Becton-Dickinson, Franklin Lakes, NJ) from an antecubital vein using 21-guage butterfly needle or from a 21-guage radial artery cannula, if that was in situ. The 10-day, 6week, and 6-month samples were collected after discharge from the hospital. For the 3- and 5-day samples, blood was collected immediately before administration of subcutaneous heparin. On collection, the blood was kept on ice for transport to the laboratory.

Within 1 hour of collection, whole blood was centrifuged for 10 minutes at $2500 \mathrm{~g}$. The plasma supernatant was then centrifuged for 10 minutes at $2500 \mathrm{~g}$, and the resultant platelet-poor plasma was stored in aliquots at $-80^{\circ} \mathrm{C}$. 


\section{Overall Hemostatic Potential}

We used a modification of the OHP assay described by $\mathrm{He}$ and colleagues. ${ }^{14,15}$ Duplicate fibrin time curves were generated in microtiter plate wells from platelet-poor plasma. Absorption measurements at 405 $\mathrm{nm}$ were taken every minute for 100 minutes. A total of 100 minutes was chosen to ensure completion of fibrinolysis. The overall coagulation potential (OCP) wells contained $75 \mu \mathrm{L}$ plasma, OHP buffer (final concentration Tris $33 \mathrm{mmol} / \mathrm{L}, \mathrm{NaCl} 65 \mathrm{mmol} / \mathrm{L}, \mathrm{CaCl}_{2} 16.5 \mathrm{mmol} / \mathrm{L}$ at $\mathrm{pH} 7.0$; tissue factor $0.85 \mathrm{pmol} / \mathrm{L}$ concentration). OHP curves were generated in a similar fashion, but the buffer also contained tissue plasminogen activator at a final concentration of $300 \mathrm{ng} / \mathrm{mL}$.

The OHP and OCP values were calculated by summation of the absorption values under the respective fibrin time curves and expressed in arbitrary units. The overall fibrinolytic potential (OFP) represented the area under the fibrinolytic portion of the curve as a proportion of the OCP $(\mathrm{OCP}-\mathrm{OHP} / \mathrm{OCP} \times 100 \%)$ and was expressed as a percentage.

\section{Statistical Analysis}

The data are presented as the mean \pm standard deviation. Normality was tested using the D' Agostino-Pearson omnibus normality test. An independent samples $t$ test, 1-way analysis of variance with Dunnett's post hoc test for multiple comparisons, 2-way repeated measures analysis of variance, and stepwise forward multivariate logistic regression analysis were used, as indicated. The data were analyzed using the Statistical Package for Social Sciences, version 18 (SPSS, Chicago, Ill). From the preliminary data, we estimated a $20 \%$ increase in OCP as a result of surgery on day 1 , with a coefficient of variation of $15 \%$ of the mean, requiring approximately 10 patients in each surgical group for a power of 0.90 and a significance of $P=.01$. We included 63 patients recruited during the 12 months of recruitment to ensure a similar patient recruitment and surgical environment during the study period.

\section{RESULTS}

The preoperative characteristics of $30 \mathrm{CABG}$ and $33 \mathrm{OP}-$ $\mathrm{CAB}$ patients and 12 healthy controls aged $>40$ years are summarized in Table 1. The in-hospital postoperative outcomes are listed in Table 2.

\section{Healthy Controls Versus Patients With Coronary Artery Disease}

The hypercoagulable OHP profile of patients with coronary artery disease (CAD) awaiting surgical revascularization

TABLE 1. Preoperative characteristics

\begin{tabular}{lccc}
\hline \multicolumn{1}{c}{ Characteristic } & CABG & OPCAB & $\begin{array}{c}\text { Healthy controls } \\
\text { aged }>\mathbf{4 0 ~ y}\end{array}$ \\
\hline Patients & 30 & 33 & 12 \\
Age (y) & $64.8 \pm 9.7$ & $65.4 \pm 10.0$ & $52 \pm 9.4$ \\
Female gender & $6(20.0)$ & $7(21.2)$ & $8(66.6)$ \\
Hypertension & $26(86.7)$ & $32(97.0)$ & $1(8.3)$ \\
Hypercholesterolemia or & $26(86.7)$ & $29(87.9)$ & $3(25)$ \\
$\quad$ statin use & & & \\
Diabetes & $11(36.7)$ & $12(36.4)$ & $2(16.7)$ \\
Smoking history & $21(70.0)$ & $17(51.5)$ & \\
Current smoker & $7(23.3)$ & $5(15.2)$ & $1(8.3)$ \\
Aspirin up to day of surgery & $9(30.0)$ & $29(87.9)$ & \\
History of MI & $14(46.7)$ & $17(56.7)$ & $0(0)$ \\
MI within 21 d & $13(43.3)$ & $12(36.4)$ & \\
Clopidogrel & $6(18.8)$ & $8(25.8)$ & \\
\hline
\end{tabular}

Data presented as $\mathrm{n}$, mean \pm standard deviation, or $\mathrm{n}(\%)$. CABG, Coronary artery bypass grafting; $O P C A B$, off-pump coronary artery bypass; $M I$, myocardial infarction.
TABLE 2. Postoperative outcomes

\begin{tabular}{lccc}
\hline \multicolumn{1}{c}{ 30-d Postoperative outcome } & CABG & OPCAB & $\boldsymbol{P}$ value \\
\hline Mortality & 0 & 0 & NS \\
Stroke & 1 & 0 & NS \\
MI & 0 & 0 & NS \\
Blood loss (mL) & $327 \pm 185$ & $326 \pm 163$ & .9885 \\
Patients transfused pRBCs (n) & 12 & 9 & .476 \\
Patients transfused other products (n) & 9 & 8 & .836 \\
Median ventilation time (h) & 10 & 14.5 & $.0128^{*}$ \\
Median ICU stay (d) & 3.3 & 2.5 & .5488 \\
Median hospital stay (d) & 6 & 6 & .9664 \\
\hline
\end{tabular}

$\overline{C A B G \text {, Coronary artery bypass grafting; } O P C A B \text {, off-pump coronary artery bypass; }}$ $M I$, myocardial infarction; $p R B C s$, packed red blood cells; $I C U$, intensive care unit. *Statistically significant.

was compared with that of healthy controls (healthy controls, $8.65 \pm 3.68$ vs patients, $13.39 \pm 4.9 ; P=.0012)$. Fibrin generation $(\mathrm{OCP})$ was elevated in the patients with $\mathrm{CAD}$ (healthy controls, $70.30 \pm 11.87$ vs patients, $87.01 \pm 13.85$; $P=.0002$ ) and fibrinolysis potential (OFP) was impaired (controls, $88.07 \% \pm 2.98 \%$ vs patients, $84.80 \% \pm 4.72 \%$; $P=.024)$.

\section{Changes in Hemostasis Early After Coronary Artery Surgery}

The patients who underwent either $\mathrm{CABG}$ or $\mathrm{OPCAB}$ demonstrated a marked exaggeration of hypercoagulable OHP parameters compared with the preoperative baseline results until at least postoperative day 10 (Figure 1, A). In both groups, fibrin generation was elevated on days 3, 5, and 10 (OCP, CABG peak day 5, $108.2 \pm 10.49$, $P=.0001$; and OPCAB peak day 3, $104.9 \pm 12.54$, $P=.0013$; Figure 2). Fibrinolysis was impaired on postoperative days 1,3 , and 5 (CABG, OFP trough on day 1 , $30.90 \% \pm 33.4 \%, P<.00001$; OPCAB, OFP trough, $66.48 \% \pm 31.31 \%, P=.0065$; Figure 3 ).

\section{Changes in Hemostasis After CABG Compared With OPCAB}

No difference was found in fibrin generation (OCP) between the $\mathrm{CABG}$ and $\mathrm{OPCAB}$ groups at any points (Figure 1, B). In contrast, fibrinolysis was significantly more impaired after $\mathrm{CABG}$ than after OPCAB on day 1 $(P=.004)$; however, by day 3 , the lower OFP after CABG compared with that after OPCAB was no longer statistically significant $(P=.053$; Figure $1, C)$. No difference was found in OFP between the 2 groups at 5 and 10 days, 6 weeks, or 6 months postoperatively.

The OHP values were significantly greater 1 and 3 days after CABG than after $\mathrm{OPCAB}(P=.011$ and $P=.035$, respectively; Figure 1). No significant difference was found in the OHP values between the CABG and OPCAB groups at the other measurement points. 

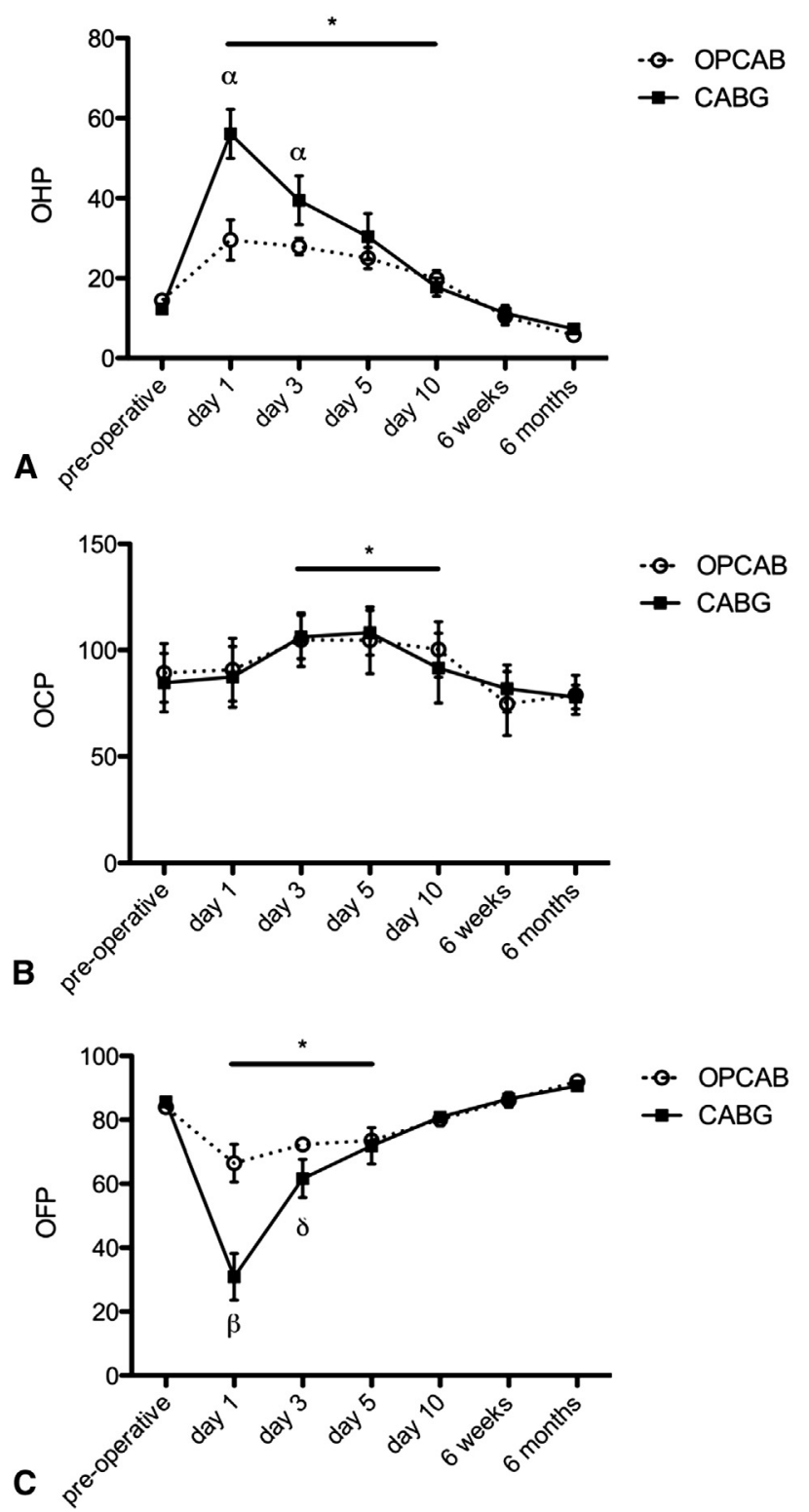

FIGURE 1. Coronary artery bypass grafting $(C A B G)$ versus off-pump coronary artery bypass $(O P C A B)$ to 6 months. A, overall hemostatic potential $(O H P)$; B, overall coagulation potential $(O C P)$; $\mathrm{C}$, overall fibrinolytic potential $(O F P) . * P<.05$ relative to preoperative baseline; $\alpha P<.05$, $\beta P<.01$, and $\delta P=.053$ between groups at indicated points.

\section{Hemostasis Late After Surgical Revascularization}

The hemostatic parameters of the patients at 6 weeks and 6 months after surgical revascularization were compared with their preoperative values and those of the healthy controls. The overall hemostatic potential did not differ significantly at 6 weeks; however, by 6 months, it was significantly lower than the preoperative values (preoperative OHP, $13.39 \pm 4.95$ vs 6-week OHP, $10.74 \pm 5.65$ $[P=.177]$ vs 6-month OHP, $6.55 \pm 2.06[P=.0074]$; Figure 2). Fibrin generation was significantly decreased 6

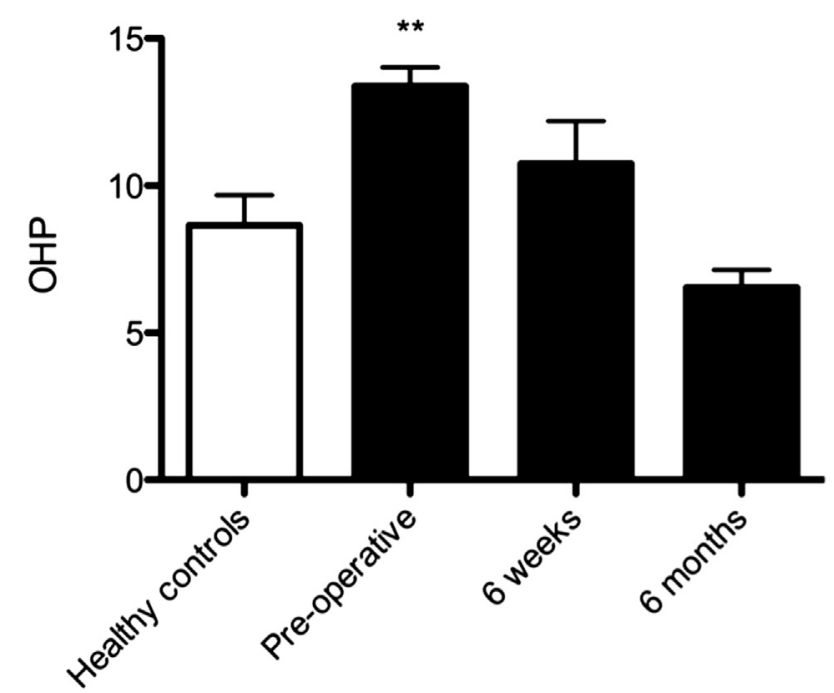

FIGURE 2. Overall hemostatic potential $(O H P)$ before and after surgical revascularization compared with healthy controls. $* * P=.0012$ compared with healthy controls.

weeks after surgery, and fibrinolysis had increased at 6 months after surgery, compared with preoperatively (preoperative OCP, $87.01 \pm 13.85$ vs 6-month OCP, $77.71 \pm 13.66$ $[P=.027]$; preoperative OFP, $84.80 \% \pm 4.72 \%$, vs 6-month OCP, $91.39 \% \pm 2.833 \%[P=.0134])$.

\section{Effect of Recent MI on Hemostasis Before Surgery}

Of the 63 patients included in the present study, 25 (13 of 33 OPCAB patients and 12 of 30 CABG patients) experienced an MI in the 21 days preceding surgical revascularization. Preoperative fibrin generation was greater in those

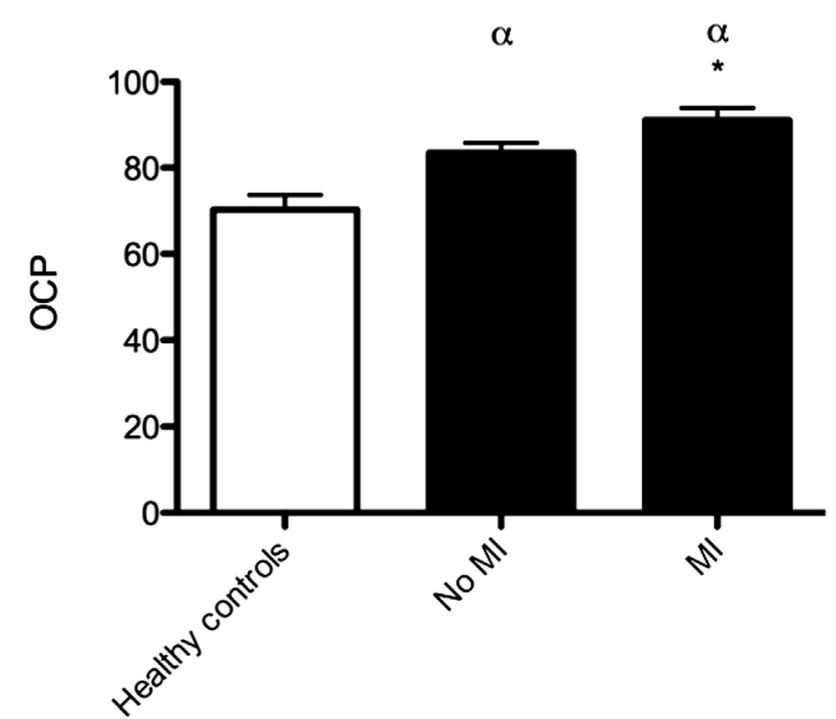

FIGURE 3. Overall coagulation potential $(O C P)$ of healthy controls versus patients with or without preoperative myocardial infarction $(M I)$. $* P<.05$, no MI versus MI; $\alpha P<.01$ compared with healthy controls. 
with a recent MI than in those without a MI (OCP, $91.07 \pm$ 14.39 vs $83.45 \pm 12.96 ; P=.0417$; Figure 3$)$. Fibrinolysis (OFP) and overall hemostasis (OHP) did not differ significantly between those with versus without MI before surgery.

\section{Effect of Preoperative MI on Hemostasis \\ Postoperatively}

The influence of MI (within 21 days of surgery) on postoperative hemostasis is demonstrated in Figure 4. In all patients, by repeated measures during 4 points to day 5 , preoperative MI influenced overall hemostatic (OHP, $P=.013)$ and fibrinolysis capacity, with the latter failing to reach statistical significance (OFP, $P=.081$ ). Preoperative MI did not influence fibrin generation (OCP, $P=.162$ ).
This pattern of postoperative hypercoagulability in patients with preoperative MI was observed only in those undergoing CABG (OHP, $P=.035$; OFP, $P=.035$; OCP, $P=.346$ ); MI did not influence the OHP, OCP, or OFP in the OPCAB patients. Stepwise multivariate analysis, including preoperative and perioperative characteristics, confirmed that a recent MI was an independent predictor of OHP 3 and 5 days postoperatively in the patient cohort when grouped together (day $3, P=.024$; day $5, P=.021$ ) and in the group that underwent CABG (day 3, $P=.049$; day $5, P=.022$ ).

In patients who had experienced a preoperative MI (more hypercoagulable than those without MI), a trend was seen for decreased postoperative blood loss that did not reach statistical significance (no MI, $392 \pm 283 \mathrm{~mL}$ vs MI, 284 $\pm 153 \mathrm{~mL} ; P=.09$ ). $\rightarrow$ NoMl
$\rightarrow$ MI
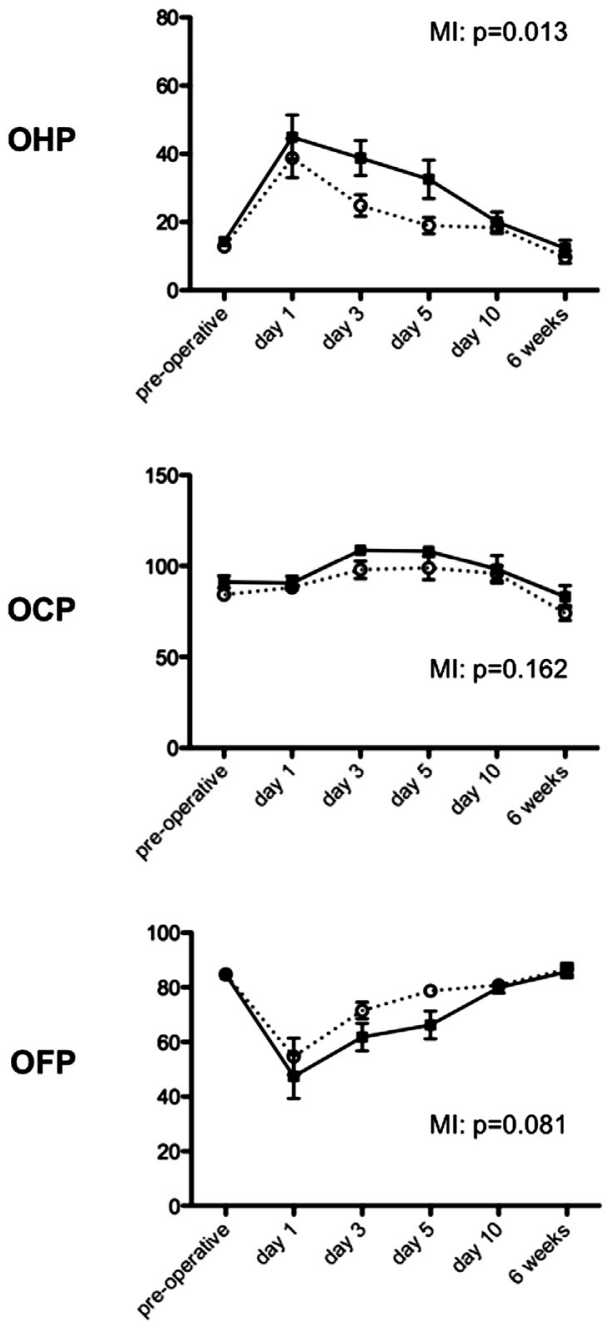

CABG
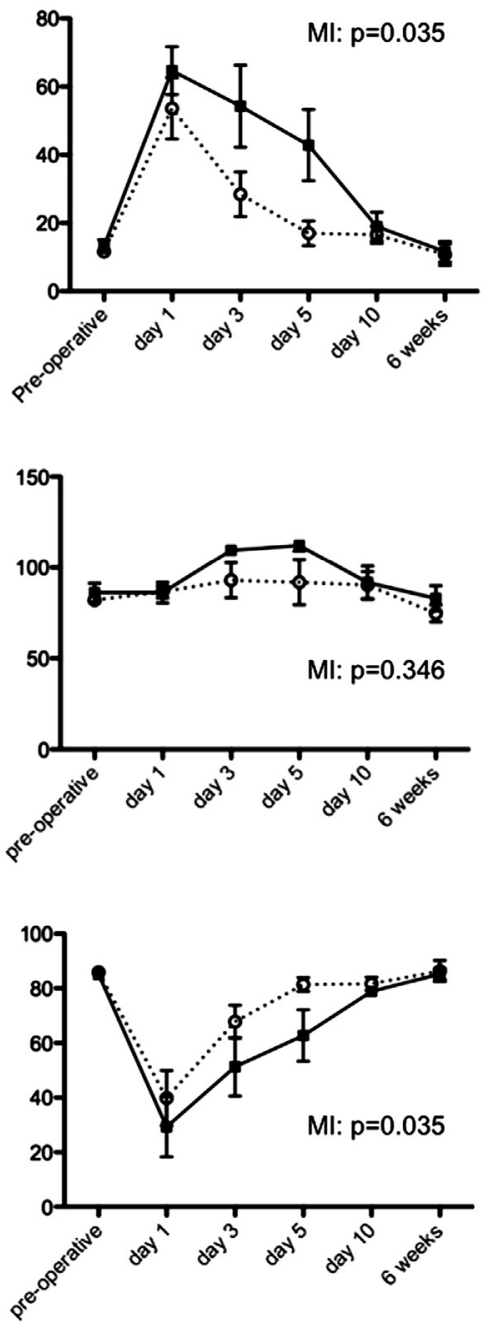

OPCAB
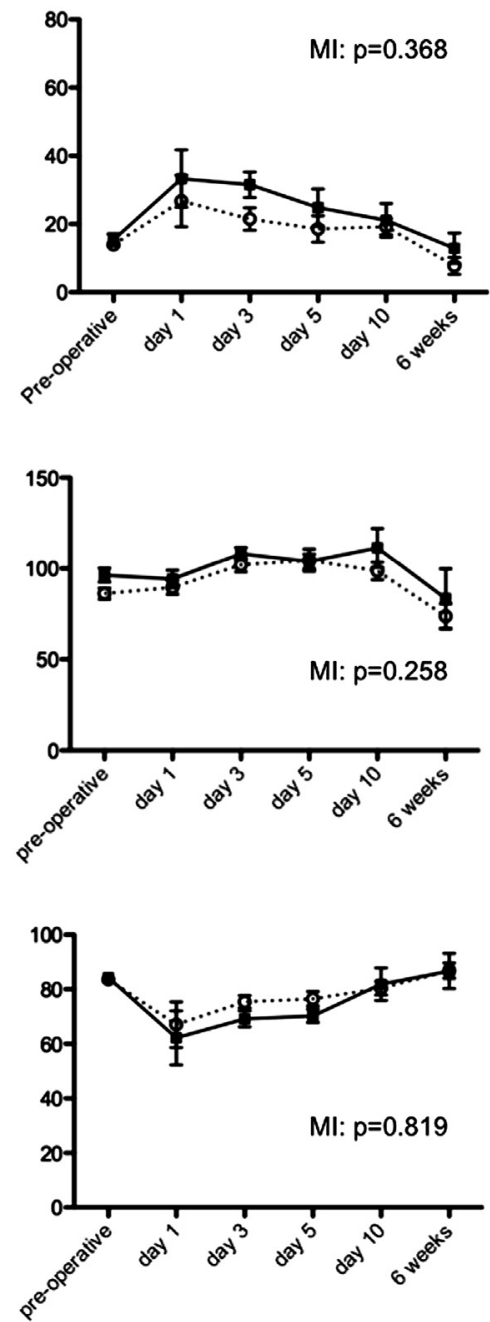

FIGURE 4. Preoperative myocardial infarction $(M I)$ versus no preoperative MI. Grouped into all patients, coronary artery bypass grafting $(C A B G)$ and offpump coronary artery bypass $(O P C A B)$, with overall hemostatic potential $(O H P)$, overall coagulation potential $(O C P)$, and overall fibrinolytic potential $(O F P)$ measured. Repeated measures analysis of variance for MI (first 4 points); $P$ values for influence of MI shown. 


\section{Influence of Other Preoperative Risk Factors on Hemostasis}

Current smokers showed an exaggeration of impaired postoperative fibrinolysis compared with non- or exsmokers (OFP day 5: smokers, $64.02 \% \pm 27.66 \%$ vs non- or exsmokers, $76.12 \% \pm 7.89 \% ; P=.047)$. This resulted in a trend of increased OHP 3 and 5 days after surgery that did not reach statistical significance. A trend was seen for decreased OFP and increased OHP in patients with diabetes on days 3 and 5 after (but not before) surgery compared with those without diabetes (OFP day 5, those without diabetes, $77.55 \% \pm 8.08 \%$ vs those with diabetes, $67.06 \% \pm$ $20.85 \%, P=.092$; OHP day $5,20.68 \% \pm 12.95 \%$ vs $31.53 \% \pm 21.95 \%, P=.098)$.

The preoperative hemostatic potentials did not differ between the patients transfused blood products after surgery and those who did not receive a transfusion. The transfused patients were older $(P=.004)$, had a longer intensive care unit and hospital stay $(P=.009$ and $P=.027$, respectively), and showed a trend toward an increased ventilation time $(P=.053)$.

\section{Role of TXA}

All CABG patients were routinely given TXA intraoperatively, and 9 of 33 OPCAB patients received intraoperative TXA. No difference was found in the amount of blood loss between those who did and did not receive TXA. In the OP$\mathrm{CAB}$ group, no difference was found in OFP (or other hemostatic parameters) between those who did and did not receive intraoperative TXA (OFP day 1, TXA, 60.26\% $\pm 37.64 \%$ vs no TXA, $68.97 \% \pm 29.12 \% ; P=.6)$, nor was any difference in blood loss found (TXA, $403 \pm 200 \mathrm{~mL}$ vs no TXA, $355 \pm 321 \mathrm{~mL} ; P=.6$ ). This difference might not have reached statistical significance owing to the small number of patients who had received TXA in the OPCAB group.

\section{DISCUSSION}

The present study used an assay of fibrin generation and fibrinolysis to characterize the changes in hemostasis seen in patients before and after coronary artery revascularization performed with and without CPB.

We have reported several novel findings, including that the individual mechanistic components of hemostasis (ie, fibrinolysis and coagulation) are altered differentially by $\mathrm{CABG}$ and OPCAB. We have demonstrated that fibrinolysis is the most important hemostatic parameter differentiating CABG and OPCAB. We have also described how a preoperative MI influences postoperative hemostasis differentially after $\mathrm{CABG}$ and OPCAB. We found that at 6 months after surgical revascularization, the patients no longer had a prothrombotic OHP profile compared with that of healthy controls.

Patients after both CABG and OPCAB demonstrated hypercoagulable hemostasis parameters until $\geq 10$ days after surgery. The elevated OHP results from both an increase in fibrin generation (OCP) and a decrease in the potential for fibrinolysis (OFP). Patients at 1 and 3 days after CABG had more hypercoagulable assay profiles available than did the OPCAB patients-this resulted from differences in OFP between groups, rather than from any difference in OCP. Our group and others have previously described evidence of elevated thrombin formation after CABG and OPCAB. Vallely and colleagues ${ }^{8}$ and Parolari and colleagues ${ }^{7}$ showed no difference in prothrombin fragments 1 and 2 (PF1.2) between CABG and OPCAB after the first 3 postoperative hours and $\leq 1$ month postoperatively. Our results have showed similarly elevated fibrin generation (OCP) after both CABG and OPCAB until postoperative day 10 and support these findings. The lack of a difference between cardiac surgery performed with or without CPB supports the limited influence of contact activation or the intrinsic pathway on postoperative hemostasis. The prolonged increase in fibrin generation (OCP) can be explained by increased plasma tissue factor described $\leq 1$ month after surgery. ${ }^{16}$ The plasma and cell-bound tissue factor were increased as a result of the surgical injury and systemic inflammation.

Measuring D-dimer and plasminogen, our group and others have described very early activation of fibrinolysis after CABG (after removal of the crossclamp). Fibrinolysis will be similarly activated 24 hours after OPCAB. ${ }^{7,8}$ When fibrin deposition is increased, an expected activation of fibrinolysis occurs. ${ }^{4}$ However, the capacity to further increase fibrinolysis, measured by OFP, could be limited and, therefore, could contribute to the hypercoagulable state. Impaired OFP might be a more useful indicator of the prothrombotic state than relative changes in the products of fibrin degradation.

Impairment of fibrinolysis has been associated with postoperative adverse thrombotic events. ${ }^{17}$ Moor and colleagues ${ }^{17}$ detected increased plasminogen activator inhibitor 1 (PAI-1) in patients with early vein graft closure. Impairment of the fibrinolytic potential after $\mathrm{CABG}$ and OPCAB could have several causes. PAI-1, an endothelial acute phase reactant, is increased 15 -fold in the first 2 hours after CABG. ${ }^{4} \mathrm{PAI}-1$ prevents the formation of plasmin and thus impairs fibrinolysis. PAI-1 and tissue plasminogen activator levels were elevated similarly after $\mathrm{CABG}$ and OPCAB until at least postoperative day $6 .{ }^{18}$ All CABG patients, but only some OPCAB patients, received TXA, which might have impaired fibrinolysis at day 1 (discussed below).

Patients awaiting coronary revascularization had prothrombotic OHP assay parameters compared with healthy controls in a pattern similar to that recently described in the Stockholm Heart Epidemiology Program. ${ }^{12}$

Our results suggest that surgical revascularization eliminates the prothrombotic profile compared with healthy 
controls in the mid- to long term. The close links between the systems of inflammation and coagulation are well established. Coronary revascularization does not remove the coronary atherosclerotic burden but, rather, directs blood to myocardial territories beyond the areas of stenosis. By improving the myocardial blood supply, ischemia is decreased; this might reduce inflammation and ameliorate the preoperative prothrombotic state, resulting in hemostatic function comparable to that of healthy controls.

MI before surgery influenced hemostasis in the postoperative period. The OHP was increased at days 3 and 5, and a trend was seen toward decreased postoperative blood loss compared with those with no MI that did not reach statistical significance. The hypercoagulability was exaggerated in patients who had undergone CABG. In these patients, a trend was seen toward decreased OFP that did not reach statistical significance, but no difference was seen in OCP.

CABG includes a period of global myocardial ischemia owing to application of an aortic crossclamp with (in our institution) the use of cold blood cardioplegia for myocardial protection. The biomarkers of myocardial injury taken from coronary sinus blood have suggested that myocardial injury can result from both myocardial ischemia and the inflammatory response to CPB. ${ }^{19,20}$ Those with preoperative MI might be more susceptible to the myocardial ischemia, reperfusion, or inflammatory response associated with $\mathrm{CPB}$. Also, the inflammation associated with myocardial injury might delay recovery of the fibrinolysis potential. Thrombin-activatable fibrinolysis inhibitor might influence fibrinolysis to a greater extent in patients with preoperative MI, because it will be elevated for at least until 7 days after $\mathrm{MI}^{21}$ and for a similar period after $\mathrm{OPCAB}^{22}$ and $\mathrm{CABG}^{23}$ compared with the preoperative baseline values.

All CABG patients and 9 of 33 OPCAB patients received intraoperative intravenous TXA, a synthetic lysine derivative that binds to plasminogen, preventing fibrinolysis. ${ }^{24}$ TXA is thought to reduce postoperative blood loss without increasing the rate of postoperative thrombosis (stroke, MI, deep vein thrombosis). ${ }^{25}$ The half-life of intravenous TXA is 2 hours; $90 \%$ of the drug is excreted by glomerular filtration at 24 hours. ${ }^{26}$ In our study, the fibrinolysis potential was more impaired after CABG than after OPCAB until day 3 . The short half-life, together with the absence of a significant difference in OFP at any point between patients receiving and not receiving TXA during OPCAB suggests that $\mathrm{CPB}$ itself might more likely be responsible. However, this conclusion was limited by the small number of patients receiving TXA in the OPCAB group.

These results have a number of clinical implications. First, patients with a preoperative MI might have less impairment of fibrinolysis potential if they undergo OPCAB, rather than CABG. Second, given the relatively prolonged postoperative period of impaired fibrinolysis in patients with preoperative MI, together with the decreased postoperative blood loss, the use of TXA in this group might not be beneficial. Third, surgical revascularization caused correction of the hypercoagulable state, which could decrease patients' future thrombotic risk. Larger clinical trials are necessary to answer these questions.

\section{Study Limitations}

The OHP assay is performed on platelet-poor plasma and thus excludes the influence of platelets on hemostasis. Changes in the number and function of platelets in the early period after CABG and OPCAB have been studied by our group and others. ${ }^{8,27}$ Activation of platelets after OPCAB might be increased in the early postoperative period. In our study, both groups received aspirin from postoperative day 1 . However, evidence has shown varying degrees of resistance to aspirin in a proportion of patients after both CABG and OPCAB, and this will influence hemostasis. ${ }^{27,28}$

Although the preoperative variables and measured preoperative hemostatic parameters were similar between the 2 groups, because the study was not randomized, the potential for selection bias could not be excluded. Similarly, the different anticoagulation and antifibrinolytic regimens used with $\mathrm{CABG}$ and $\mathrm{OPCAB}$ made it difficult to completely isolate the role of CPB itself in hemostasis. Rather, the present study compared the hemostatic parameters of the procedures as a whole (not just the avoidance of CPB).

\section{CONCLUSIONS}

Patients after both $\mathrm{CABG}$ and $\mathrm{OPCAB}$ remained in a prothrombotic state until $\geq 10$ days after surgery. This resulted from both an increase in coagulation and impairment of fibrinolysis potential. Patients had a more prothrombotic profile on days 1 and 3 after CABG, relative to OPCAB. Patients with CAD have prothrombotic OHP assay parameters before surgery; however, by 6 months after revascularization, they will have a hemostatic profile comparable to that of healthy controls. A preoperative MI influences postoperative hemostasis and might warrant an individual approach to management. Consideration should be given to using $\mathrm{OPCAB}$ or increasing prophylactic anticoagulation with medications that act on the plasma hemostatic systems (eg, heparin). Additional studies are required to clarify whether extending (and perhaps increasing) prophylactic anticoagulation for a period beyond a patient's discharge will favorably affect the incidence of thrombotic complications in patients undergoing surgical revascularization.

\section{References}

1. Warren OJ, Smith AJ, Alexiou C, Rogers PLB, Jawad N, Vincent C, et al. The inflammatory response to cardiopulmonary bypass: part 1-mechanisms of pathogenesis. J Cardiothorac Vasc Anesth. 2009;23:223-31.

2. Boisclair MD, Lane DA, Philippou H, Esnouf MP, Sheikh S, Hunt B, et al. Mechanisms of thrombin generation during surgery and cardiopulmonary bypass. Blood. 1993;82:3350-7. 
3. De Somer F, Van Belleghem Y, Caes F, François K, Van Overbeke H, Arnout J, et al. Tissue factor as the main activator of the coagulation system during cardiopulmonary bypass. J Thorac Cardiovasc Surg. 2002;123:951-8.

4. Sniecinski RM, Chandler WL. Activation of the hemostatic system during cardiopulmonary bypass. Anesth Analg. 2011;113:1319-33.

5. Raja SG, Berg GA. Impact of off-pump coronary artery bypass surgery on systemic inflammation: current best available evidence. J Card Surg. 2007;22:445-55.

6. Edelman JJ, Yan TD, Bannon PG, Wilson MK, Vallely MP. Coronary artery bypass grafting with and without manipulation of the ascending aorta-a meta-analysis. Heart Lung Circ. 2011;20:318-24.

7. Parolari A, Mussoni L, Frigerio M, Naliato M, Alamanni F, Galanti A, et al, Increased prothrombotic state lasting as long as one month after on-pump and off-pump coronary surgery. J Thorac Cardiovasc Surg. 2005;130:303-8.

8. Vallely MP, Bannon PG, Bayfield MS, Hughes CF, Kritharides L. Quantitative and temporal differences in coagulation, fibrinolysis and platelet activation after on-pump and off-pump coronary artery bypass surgery. Heart Lung Circ. 2009; 18:123-30.

9. Kolh P, Kerzmann A, Lahaye L, Gerard P, Limet R. Cardiac surgery in octogenarians: peri-operative outcome and long-term results. Eur Heart J. 2001;22: 1235-43.

10. Shahian DM, O'Brien SM, Filardo G, Ferraris VA, Haan CK, Rich JB, et al. Report of STS Quality Measurement Task Force: The Society of Thoracic Surgeons 2008 cardiac surgery risk models: part 1—coronary artery bypass grafting surgery. Ann Thorac Surg. 2009;88:S2-22.

11. Roques F, Nashef SA, Michel P, Gauducheau E, de Vincentiis C, Baudet E, et al. Risk factors and outcome in European cardiac surgery: analysis of the EuroSCORE multinational database of 19030 patients. Eur J Cardiothorac Surg. 1999; 15:816-22.

12. Leander K, Blombäck M, Wallén H, He S. Impaired fibrinolytic capacity and increased fibrin formation associate with myocardial infarction. Thromb Haemost. 2012;107:1092-9.

13. Curnow JL, Morel-Kopp M-C, Roddie C, Aboud M, Ward CM. Reduced fibrinolysis and increased fibrin generation can be detected in hypercoagulable patients using the overall hemostatic potential assay. J Thromb Haemost. 2007:5:528-34.

14. He S, Antovic A, Blombäck M. A simple and rapid laboratory method for determination of hemostasis potential in plasma. II. Modifications for use in routine laboratories and research work. Thromb Res. 2001;103:355-61.

15. He S, Bremme K, Blombäck M. A laboratory method for determination of overall hemostatic potential in plasma. I. Method design and preliminary results. Thromb Res. 1999;96:145-56.

16. Parolari A, Mussoni L, Frigerio M, Naliato M, Alamanni F, Polvani GL, et al. The role of tissue factor and P-selectin in the procoagulant response that occurs in the first month after on-pump and off-pump coronary artery bypass grafting. $J$ Thorac Cardiovasc Surg. 2005;130:1561-6.
17. Moor E, Blombäck M, Silveira A, Wiman B, Cederlund K, Bergstrand L, et al Hemostatic function in patients undergoing coronary artery bypass grafting: perioperative perturbations and relations to saphenous vein graft closure. Thromb Res. 2000;98:39-49.

18. Paparella D, Galeone A, Venneri MT, Coviello M, Scrascia G, Marraudino N, et al. Activation of the coagulation system during coronary artery bypass graft ing: comparison between on-pump and off-pump techniques. J Thorac Cardiovasc Surg. 2006;131:290-7.

19. Rastan AJ, Bittner HB, Gummert JF, Walther T, Schewick CV, Girdauskas E, et al. On-pump beating heart versus off-pump coronary artery bypass surgeryevidence of pump-induced myocardial injury. Eur J Cardiothorac Surg. 2005; 27:1057-64.

20. Narayan P, Rogers CA, Bayliss KM, Rahaman NC, Panayiotou N, Angelini GD et al. On-pump coronary surgery with and without cardioplegic arrest: comparison of inflammation, myocardial, cerebral and renal injury and early and late health outcome in a single-centre randomised controlled trial. Eur J Cardiothorac Surg. 2011;39:675-83.

21. Shantsila E, Montoro-García S, Tapp LD, Apostolakis S, Wrigley BJ, Lip GYH Fibrinolytic status in acute coronary syndromes: evidence of differences in relation to clinical features and pathophysiological pathways. Thromb Haemost. 2012;108:32-40.

22. Paparella D, Semeraro F, Scrascia G, Galeone A, Ammollo CT, Kounakis G, et al Coagulation-fibrinolysis changes during off-pump bypass: effect of two heparin doses. Ann Thorac Surg. 2010;89:421-7.

23. Lisowski P, Małyszko J, Hirnle T, Lisowska A, Jackowski R, Małyszko JS, et al Thrombin activatable fibrinolysis inhibitor (TAFI) in stable angina pectoris patients undergoing coronary artery bypass grafting (CABG). Rocz Akad Med Bialymst. 2005;50:166-72.

24. McCormack PL. Tranexamic acid: a review of its use in the treatment of hyperfibrinolysis. Drugs. 2012;72:585-617.

25. Henry DA, Carless PA, Moxey AJ, O'Connell D, Stokes BJ, Fergusson DA, et al Anti-fibrinolytic use for minimising perioperative allogeneic blood transfusion. Cochrane Database Syst Rev. 2011;CD001886.

26. Fiechtner BK, Nuttall GA, Johnson ME, Dong Y, Sujirattanawimol N, Oliver WC, et al. Plasma tranexamic acid concentrations during cardiopulmonary bypass. Anesth Analg. 2001;92:1131-6.

27. Bednar F, Osmancik P, Vanek T, Mocikova H, Jares M, Straka Z, et al. Platelet activity and aspirin efficacy after off-pump compared with on-pump coronary artery bypass surgery: results from the prospective randomized trial PRAGUE 11 - Coronary Artery Bypass and REactivity of Thrombocytes (CABARET). $J$ Thorac Cardiovasc Surg. 2008;136:1054-60.

28. Zimmermann N, Kurt M, Wenk A, Winter J, Gams E, Hohlfeld T. Is cardiopulmonary bypass a reason for aspirin resistance after coronary artery bypass grafting? Eur J Cardiothorac Surg. 2005;27:606-10. 